Historic, Archive Document

Do not assume content reflects current scientific knowledge, policies, or practices. 



\section{NEW Price List, March, 1929}

\section{DESTROY PREVIOUS LIST \\ CHAMPLAIN VIEW GARDAdifre: \\ ELMER E. GOVE \\ Burlington, Vermont \\ *. MAR J 11929 \\ U. B. Le baruse et of A gricaltomes.}

At these prices all bulbs are prepaid anywhere in the United. Stard countries in the Postal Union.

The PACKAGES of Bulblets contain from fifty to one hundred or more bulblets. The cheaper varieties contain more than the others. They are not counted but all are big value.

For TERMS, EXTRAS, Etc., see page 2 of the Catalog.

5 at 10 rate; 25 at 100 rate; 250 at 1000 rate. Bulblets, 250 at 1000 rate.

\begin{tabular}{|c|c|c|c|c|c|c|c|c|c|}
\hline E. Kunderd....... & $\begin{array}{r}P e r \\
1 \\
10\end{array}$ & $\begin{array}{l}\text { No.1 } \\
\$ 1.25 \\
10.00\end{array}$ & $\begin{array}{r}\text { No. } 2 \\
\$ 1.00 \\
8.00\end{array}$ & $\begin{array}{r}\text { No.3 } \\
\$ .75 \\
6.00\end{array}$ & $\begin{array}{r}\text { No. } 4 \\
\$ .60 \\
4.75\end{array}$ & $\begin{array}{r}N o .5 \\
\$ .45 \\
3.80\end{array}$ & $\begin{array}{r}\text { No. } 6 \\
\$ .25 \\
2.00\end{array}$ & $\begin{array}{r}\text { Per } \\
10 \\
100\end{array}$ & $\$ 4.5$ \\
\hline banis & & $\begin{array}{l}.10 \\
.75\end{array}$ & .65 & $\begin{array}{l}.07 \\
.55\end{array}$ & .45 & .35 & .25 & Pkg. & \\
\hline & $\begin{array}{r}100 \\
1000\end{array}$ & 4.00 & $\begin{array}{r}3.00 \\
27.00\end{array}$ & $\begin{array}{r}2.00 \\
18.00\end{array}$ & ..... & …-. & - & $\begin{array}{l}1000 \\
\text { Qt. }\end{array}$ & \\
\hline atross. & 1 & 3.50 & 3.00 & 2.50 & (......... & .......... & ............... & ..... & \\
\hline & 1 & .10 & & .07 & & ...... & $\ldots . .$. & $\mathrm{kg}$. & \\
\hline & $\begin{array}{r}10 \\
100\end{array}$ & …..... & $\begin{array}{r}.55 \\
4.00\end{array}$ & $\begin{array}{r}.45 \\
3.20\end{array}$ & $\begin{array}{r}.35 \\
2.50\end{array}$ & ............ & $\ldots$ & $\begin{array}{l}1000 \\
\text { Qt. }\end{array}$ & $4 .($ \\
\hline horet & 1 & .50 & .40 & .30 & .20 & .15 & .10 & Pkg. & \\
\hline & 10 & & & 2.50 & 1.60 & 1.20 & .80 & & \\
\hline igel's Drea & 1 & 10.00 & 8.50 & 6.00 & 4.50 & ......... & ............ & ..... & \\
\hline . & $\begin{array}{r}1 \\
10\end{array}$ & $\begin{array}{l}.10 \\
.60\end{array}$ & .50 & $\begin{array}{l}.07 \\
.40\end{array}$ & .30 & .20 & .10 & $\begin{array}{l}\text { Pkg. } \\
1000\end{array}$ & \\
\hline & 100 & & 4.00 & 3.20 & 2.40 & 1.60 & 80 & Qt & \\
\hline ina Eberius & 1 & .10 & & .07 & & 30 & ......... & Pkg. & \\
\hline & 10 & .60 & .50 & .40 & .30 & .20 & $\ldots+.$. & 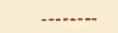 & \\
\hline nnie Laurie. & 1 & .35 & .30 & .25 & .20 & .15 & .10 & 10 & 2. \\
\hline & 100 & 25.00 & 20.00 & $\begin{array}{r}2.00 \\
15.00\end{array}$ & 12.00 & 10.00 & 8.00 & 1000 & 15. \\
\hline $20 \%$ off & $\{1000$ & & 160.00 & 120.00 & .00 & 80.00 & 640 & & \\
\hline nthony Zon & 1 & ........ & $\ldots$ & .10 & $\ldots$ & ...... & .......... & Pkg. & \\
\hline & 10 & 2.00 & $\begin{array}{r}.90 \\
1.75\end{array}$ & $\begin{array}{l}.80 \\
50\end{array}$ & $\begin{array}{r}.60 \\
1.00\end{array}$ & $\begin{array}{l}.50 \\
75\end{array}$ & .40 & ach & \\
\hline 2 AIIUU & 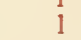 & .30 & .25 & .20 & .15 & 10 & $\cdots$ & 10 & \\
\hline & 10 & 2.40 & 2.00 & 1.60 & 1.20 & .80 & .60 & 100 & .1 \\
\hline$\ldots \ldots$ & 1 & $\begin{array}{l}25 \\
20\end{array}$ & .20 & .15 & ......... & .10 & -........ & Pkg. & \\
\hline 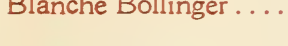 & 10 & $\begin{array}{l}.20 \\
1.60\end{array}$ & 120 & .10 & 60 & 40 & 25 & $\begin{array}{l}\text { Pkg. } \\
1000\end{array}$ & \\
\hline & 1 & .10 & & .07 & & & & & \\
\hline Hea & 1 & 4.00 & 3.50 & 3.00 & 2.50 & 2.00 & 1.50 & 10 & \\
\hline Torch. & 1 & .35 & .30 & .25 & .20 & .15 & .10 & Pkg. & \\
\hline & $\begin{array}{r}10 \\
100\end{array}$ & 280 & 2.4 & $\begin{array}{r}2.00 \\
12.00\end{array}$ & $\begin{array}{r}1.60 \\
10.00\end{array}$ & $\begin{array}{l}1.20 \\
6.00\end{array}$ & $\begin{array}{r}.80 \\
5.00\end{array}$ & & \\
\hline $\mathrm{bb}$ & 1 & .30 & .25 & .20 & .15 & .10 & & 0 & \\
\hline & 1 & & 2.00 & 1.60 & 1.20 & .75 & .60 & 1000 & \\
\hline & 1 & .15 & .......... & .10 & & .......... & .......... & Pkg. & \\
\hline eil & 1 & 1.50 & 1.50 & 1.00 & 1.00 & ...... & $\cdots$ & Each & \\
\hline n L. Smith...... & 1 & -....... & .1 & .07 & ... & 3 & 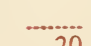 & Pkg. & \\
\hline sernet. & 1 & 2.50 & 2.00 & $\begin{array}{r}.50 \\
1.60\end{array}$ & 1.25 & 1.00 & .75 & 10 & 2. \\
\hline
\end{tabular}


$\begin{array}{llllll}\text { Per No.1 No.2 No.3 No.4 No.5 No.6 } & \text { No. }\end{array}$

Bulblets

100

Cardinal Prince...... 1

10

100

1000

Car. Clusius........ 1

10

Catherine Coleman...

Chas. F. Eairbanks... 10

Chicago

Circe.............

Coryphee. ........

Crimson Glow ........

100

Crinkles........... 1

Desdemone......... 1

10

Diana............ 1

Diener's Amer. Beauty.. 1

Don Juan. ..........

Dorcas Aldrich...... 1

Dorine............ 1

Dorritt.......... 1

Dr. Christ Martz ....

Dr. Elkins........

10

Dr. F. E. Bennett.... 1

Dr. Moody...........

Dr. J. H. Neeley.....

Dr. R. T. Jackson.... 1

Dr. W. Van Fleet....

Duchess of York..... 1

Early Phipps........

Ed. Springer......... $\begin{array}{rr}.10 & . . .1 . \\ .80 & .70 \\ 7.00 & 6.00\end{array}$

.07

.25

2.00

12.00

2.00

18.00

.30

2.50

.15

.90

7.50

.10

.75

7.00

.10

$.60 \quad .50$

$\begin{array}{rr}. . . & 3.00 \\ \cdots-. . & 20.00 \\ . .20\end{array}$

1.50

.25

2.00

.10

.60

1.25

$\begin{array}{ll}. . . . & .10 \\ . . . & .65\end{array}$

$.25 \quad .20$

1.00

$\begin{array}{lll}.80 & .60 & .50\end{array}$

$\begin{array}{ll}. . . . & .10 \quad .07\end{array}$

$\begin{array}{lll}\ldots & .10 \\ \cdots . . . . & .07\end{array}$

.25

2.00

.10

.80

.25

2.00

4.00

.20

1.60

1.20

.65

6.00

.07

5.00

.15

1.75

12.00

1.50
8.00

2.50

$\begin{array}{rrr}4.00 & 3.00 & 2.50 \\ 30.00 & 25.00 & 20.00\end{array}$

.10

.60

4.00

.10

.60

.10

.60

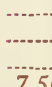

7.50
.50

3.00

.07

2.25

.07

.50

3.00

3.00

.15

.80

6.25

.40

.40

2.50

.07

.40

2.00

.10
65

5.00

.35
.50

.10
50

3.00

.40

.30

.10

.80

6.50

55.00

1.25

10.00

80.00

.15

1.50

.. .50

2.50

.60

$5.00 \quad 3.50$

$45.00 \quad 30.00$

$1.00 \quad .75$

$8.00 \quad 6.00$

$70.00 \quad 50.00$

.12

1.25

.85

.40

.30

$\ldots$

3.00

.30

......................

$\begin{array}{ll}2.50 & 2.00\end{array}$

.30
1.50

$.20 \quad .15$

10.00

.85

…-....

.65

$.10 \quad .08$

.65

.50
.55

.30

.20

.15

45

.35

.25

.........

.30

$-.10$

.20

$-.80$

.45

4.00

.10

1.25

6.00

2.00

15.00

.60

.35

3.00

1.00

4.00

1.50

10.00

.30

...............

1.75

30

$-\cdots$
-......

$$
1.50
$$

.45

4.00

.30
Per

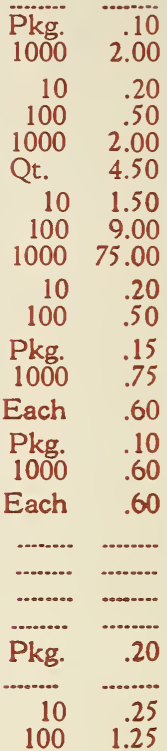

Pkg. $\quad .10$

$1000 \quad .80$

Pkg. $\quad .10$

$1000 \quad .80$

Pkg. .25

$10 \quad .50$

Pkg. $\quad .10$

Pkg. $\quad .20$

$1000 \quad 1.50$

Pkg. $\quad .10$

$1000 \quad 1.00$

Qt. $\quad 4.00$

$10 \quad .15$

$100 \quad 350$
$1000 \quad 3.50$

3.00

1.00

8.00

$10 \quad 3.50$

$100 \quad 18.00$

Pkg. $\quad .10$

Qt. $\quad 2.00$

Pkg. $\quad .10$

1000.50

Qt. $\quad 1.50$

Pkg. $\quad .10$

$1000 \quad .50$

Qt. $\quad 1.50$

Pkg. $\quad .15$

$1000 \quad 1.00$

Each .50

$10 \quad .40$ 
Per No.1 No. 2 No.3 No.4 No. 5 No. 6

E. G. Hill........ $\begin{array}{r}1 \\ 10 \\ 100\end{array}$

E. J. Shaylor....... 1

Elf........... 1

10

100

Elizabeth Tabor 1000

10

100

Ella Day.............

Emile Aubrun.......

1
1

10

100

1000

Ensign............ 1

Excello........... 1

Fay Lamphier.......

Fern Kyle..........

10

100

Flambeau..........

Flaming Sword......

10

Fontaine..........

100

Frank M. Schick..... 10

Gallant Leader. . . . . .

Georgia..............

Gertrude Errey

$\begin{array}{r}.10 \\ .65 \\ . .10 \\ .65 \\ .10 \\ .55 \\ -.-. \\ \hline\end{array}$

25.00

Gertrude Pfitzer.....

Giant Nymph.

10

100

1000

Gloriana

100

Gloriosa

10

Gold

10

100

Gold Eagle

100

100

$20 \%$ off

10

$\left\{\begin{array}{r}100 \\ 1000\end{array}\right.$ \begin{tabular}{rr}
$\ldots . .35$ & .07 \\
3.20 & .45 \\
$\ldots .50$ \\
.55 & .07 \\
$\ldots . .45$ \\
. .45 & .07 \\
3.20 & .35 \\
\hline .05
\end{tabular}

.35
2.00
.35
$.3 . . .3$
.25
2.00

$25.00 \quad 20.00$

16.00

.10

.55

4.00

5.00

.50

4.50

1.00

.10

.65

.15

1.00

.10

.80

6.00

.45

3.20

.07

.25

2.50

$4.50 \quad 3.00$

2.00

2.00

.40

.30

$3.00 \quad 2.40$

200.00

20.00

.25

2.00

15.00

200.00

60.00

120.00

.50

.15
1.00

8.00

.10

....

.10

3.00

17.50

5.00

.50

.15

10.00

5.00

.10

5.00

$$
5.00
$$

.50

$$
4.00
$$

$$
.10
$$

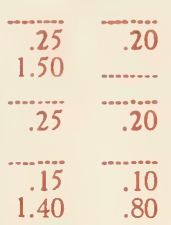

$11.00 \quad 6.00$

$\begin{array}{ll}.15 & .10\end{array}$

$1.00 \quad .80$

$1.50 \quad 1.00$

$.20 \quad .15$

$1.60 \quad 1.20$

$10.00 \quad 8.00$

$80.00 \quad 60.00$

........

.55

.07

.45

.35

.10

.80

.45

….... $\quad .07$

5.00

4.00

3.45

2.35

.25

.20

.80

6.00

.5

......

.35

.10

5.00

45

.35

4.00

.35

2.00

2.50

15.00

5.00

.40

1.00

8.00

4.00

........

.80

35.00

35.00
.40

3.50

.............

$.70 \quad .60$

$.10 \quad \cdots .-.65$

.7 .20
$.40 \quad .35$

$3.50 \quad 3.00$

$\begin{array}{rr}. .1 . & 25.00 \\ .75 & .65\end{array}$

$6.00 \quad 5.00$

50.00

40.00 .

.07

.45

2.00

1.75

8.00

.35

.10

.85

........................

6.50

5.00

$3.00 \quad 2.50$

.07

-........

.60

.45

3.00
27.00

20.00

.35

20.00
.25

3.00
18.00

2.25

2.50

.15

$.35 \quad .25$

.25
1.25

.35

1.50

6.00

……. .........

.55

4.00

2.00

..........

1.50

13.50

.15

$$
1.35
$$

8.00

.25

.07

.........

.30

.07

.55

.45

.3

$\begin{array}{lll}2.50 & 2.00 & 1.50\end{array}$

.20

.50

.25

$2.50 \quad 2.00$

1.50

$\begin{array}{rrr}20.00 & 16.00 & 12.00 \\ 55 & .45 & 35\end{array}$

$\begin{array}{lll}4.00 & 3.25 & 2.50\end{array}$

2.50

$\begin{array}{llll}32.00 & 26.00 & 20.00 & 16.00\end{array}$ $200.00 \quad 150.00 \quad 125.00$

BulbLets

Per

Pkg. $\quad .10$

$1000 \quad 1.00$

Qt. $\quad 2.50$

Pkg. $\quad .10$

$1000 \quad .50$

Pkg. $\quad .10$

$1000 \quad .50$

Qt. $\quad 1.50$

Pkg. $\quad .10$

$1000 \quad .75$

Qt. $\quad 3.00$

Each $\quad .50$

$10 \quad .20$

$100 \quad 1.00$

$1000 \quad 5.00$

Qt. $\quad 17.50$

$10 \quad 1.00$

Pkg. $\quad .10$

$1000 \quad .50$

Pkg. $\quad .10$

Pkg. $\quad .10$

$1000 \quad 2.00$

Qt. $\quad 5.00$

$10 \quad .50$

Pkg. $\quad .10$

$1000 \quad 2.50$

Qt. $\quad 5.00$

Pkg. $\quad .10$

$1000 \quad .80$

Qt. $\quad 3.00$

Pkg. $\quad .10$

$1000 \quad 1.00$

$5 \quad 1.00$

$100 \quad 5.00$

$10 \quad .50$

$10 \quad .15$

$100 \quad .50$

$1000 \quad 4.00$

Each $\quad .40$

$10 \quad 3.50$

Pkg. $\quad .10$

$1000 \quad 1.00$

Qt. $\quad 3.50$

$10 \quad .20$

$100 \quad 1.25$

$1000 \quad 8.00$

Pkg. $\quad .10$

$1000 \quad 1.50$

Pkg. $\quad .10$

$1000 \quad .75$

$\begin{array}{lr}\text { Qt. } & 3.00 \\ 10 & 50\end{array}$

$100 \quad 3.50$

$1000 \quad 30.00$

$100 \quad 3.50$

$\begin{array}{rr}100 & 3.50 \\ 1000 \quad 30.00\end{array}$

Qt. $\quad 150.00$ 
$\begin{array}{llllll}\text { Per No.1 No. } 2 & \text { No.3 } & \text { No. } 4 & \text { No. } 5 & \text { No. } 6\end{array}$

Bulblets

$\mathrm{Per}$

Golden Measure.....

Golden Salmon......

$10 \quad .10$

.07

$\begin{array}{rrrr}.07 & \cdots & & \\ .60 & .50 & .35 & . . .25 \\ .25 & .20 & .15 & .10 \\ 2.00 & 1.50 & 1.00 & .75\end{array}$

Pkg. $\quad .10$

$\begin{array}{lll}1 & .35 & .30\end{array}$

$\begin{array}{rr}.35 & .30 \\ 3.00 & 2.50\end{array}$

.50

(1.00

-........

Hamburg Pink.......

Heavenly Blue.......

Heinrich Kanzleiter. .

10
10
100

Helen Howard. . . . . 1

Helen Phipps. ...... 1

2.00

\begin{tabular}{lr}
$7.50 \quad 6.50$ \\
\hline
\end{tabular}

5.00

4.00

3.00

2.25

55.00

45.00

36.00

27.00

20.00

$\begin{array}{rr}.25 & .20 \\ 2.00 & 1.60\end{array}$

.15
1.20
10.00

.10
80

.60

.40

$15.00 \quad 12.00$

7.00

5.00

3.00

$1.00 \quad .75$

.60

.50

.40

.35

$3.00 \quad 2.50$

4.75

4.00

3.20

2.50

$25.00 \quad 20.00$

1.75

$12.00 \quad 10.00$

8.00

Helen Wills.........

1.25

1.00

.80

$.70 \quad .60$

.45

Henry Ford........ 1

100

Henry C. Goehl......

$\begin{array}{cr}1 . \cdots . . . & .10 \\ \cdots \cdots . . . & .50\end{array}$

.07

.40
3.00

.30

.20

.15

$.20 \quad .15$

10

$2.25 \quad 1.50$

1.00

.90

.75

.60

.50

7.00

5.50

4.50

3.50

Herbstzauber. ...... 1

2.50

2.00

.30

1.00

16.00

12.00

10.00

7.50

Hermes.......... 1

.10

.15

....

.60

3.50

.40

.30

Highland Laddie. .... $\quad 1 \quad .15$

-..-....

10

.80

.70

.60

.50

2.00

1.25

High Nye........ 1

$.35 \quad .30$

.25

.20

.40

.30

$\begin{array}{ll}2.50 & 2.00\end{array}$

1.50

1.00

.15

6.80

.10

.15

Illuminator......... 1

Imperator..........

Indian Summer...... 1

Iwa.............

10

100

J. A. Carbone....... 1

J. V. Beijeren.......

10
100

Jap Lady ..........

10.00

4.00

1.00

.10

.25

.25

.20

..........

.60

.50

...........

.20

.15

.12

.10

$\ldots$

$.25 \quad .20$

2.00

1.60

.15

.90

.80

1.20

.80

.60

3.50

.10
.75

5.00

.25

$.10 \quad \ldots . . . . .$.

.75

6.00

5.00

.07

.45

.35

.07

Jenny Lind..........

1
10
100

Joerg's White........

.50

4.00

... .30

.30

.15

2.00

1.00

.07

.20

$\ldots . . .1$.

.. .15

4.00

.40

1.25

$60 \quad 50$

.45

.35

.30

.20

J. O. Armour........

$.25 \quad .20$

1.20

.10

.60

.40

$.25 \quad .20$

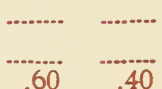

1.00

.80

1.50

1.00

$10 \quad .35$

$100 \quad 1.50$

Each .10

Each $\quad .60$

$10 \quad 5.00$

$10 \quad .25$

$100 \quad 1.25$

$1000 \quad 8.00$

$10 \quad 1.00$

$100 \quad 8.00$

Each .30

$\begin{array}{ll}10 & 2.50\end{array}$

$10 \quad 1.00$

Pkg. $\quad .10$

$1000 \quad .80$

Qt. $\quad 4.00$

Pkg. $\quad .15$

$1000 \quad 1.00$

$10 \quad .50$

$1000 \quad 15.00$

Pkg. $\quad .10$

$1000 \quad 1.00$

Qt. $\quad 2.50$

Pkg. $\quad .10$

$1000 \quad 1.00$

$10 \quad .10$

$100 \quad .70$

$1000 \quad 6.00$

Pkg. $\quad .15$

$1000 \quad 1.50$

No bulblets

$100 \quad .50$

$1000 \quad 2.00$

Pkg. .25

$1000 \quad 3.00$

Qt. $\quad 15.00$

Pkg. $\quad .10$

$1000 \quad 1.00$

Pkg. $\quad .10$

$1000 \quad .80$

No bulblets

Pkg. $\quad .10$

$1000 \quad 1.00$

Qt. $\quad 5.00$

$10 \quad .45$

$10 \quad .10$

$100 \quad .30$

$10 \quad 35$

Pkg. $\quad .10$

$1000 \quad 1.00$

King Tut.........

$\begin{array}{lll}5.00 & 4.00 \quad 3.00\end{array}$

2.00

Each

.50 
$\begin{array}{llllll}\text { Per No. } 1 & \text { No. } 2 & \text { No. } 3 & \text { No. } 4 & \text { No. } 5 & \text { No. } 6\end{array}$

BUI.BLI:TS

I'er

Kirch's Apple Blossom

Kund's Apple Blossom. .

10

Kund's Yellow Favorite

Kund's Yellow Wonder.

10

Lacinatus.........

La Paloma..........

Lauretta...........

Lilac Glory.........

Lilac Wonder........

Lily of Gold. . . . . . . .

Llewellyn...........

London Smoke.......

Longfellow.........

10

10

10
100

Los Angeles........ 1

Louvain...........

Loyalty. .

Mme. Mounet-Sully. .

Mammoth White....

Marie Kunderd...... .

Marietta.

Marion Koch........ 1

Marmora..........

$$
10
$$

100

Marnia

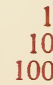

100
1000

Marshall Foch.......

1
10
100
1000

Mary Frey

$\begin{array}{rrr}.40 & .30 & .25 \\ .15 & \ldots & .10 \\ 1.25 & 1.00 & .75 \\ 8.00 & 7.00 & 6.00\end{array}$

30

20

.25

2.00

1.50

.50

4.00

.20

3.20

17.50

10.00

.15

1.25

.10

$10.00 \quad 10.00$

.50

.15

1.20

.25

2.00

$\cdots \cdots \cdots . . . . . .25$

.15

1.20

1.00

....... $\quad .25$

2.00

.25

.25

1.80

.10

.20

1.60

14.50

.70

.60

.35

3.00

2.00

.35

4.00

10.00

.10

.70
4.50

.15

-.......

1.50

2.50

$20.00 \quad 18.00$

-.......

.20

1.25

80.00

$$
.15
$$

1.00

8.00

64.00

.10

.75

6.00
48.00

48.00

.55

$4.50 \quad 3.50$

28.00

$.60 \quad .45$

4.50

3.50

$\begin{array}{ll}.15 & .10 \\ 1.00 \quad .75\end{array}$

$.20 \quad .15$

$1.60 \quad 1.20$

$14.00 \quad 10.00$

.75

.25

.70

.10

.75

$25.00 \quad 20.00$

.80

70

.15

1.20
7.20

7.20

1.20

10.25

35

.20

…-...

.10

$.70 \quad .55$

$\begin{array}{rrr}4.00 & 3.20 & 2.60 \\ & 27.00 & 20.00\end{array}$

.35

.20

$\begin{array}{rr}2.00 & 1.50 \\ 15.00 & 10.00\end{array}$

.80

$\begin{array}{llllll}6.00 & 5.00 & 4.00 & 3.00 & 2.00 & 1.50\end{array}$

$50.00 \quad 40.00$

40.00

32.00

$\begin{array}{rrr}24.00 & 16.00 & 12.00\end{array}$

\begin{tabular}{|c|c|c|}
\hline & 10 & \\
\hline & $\begin{array}{l}\mathrm{Pkg} . \\
1000\end{array}$ & \\
\hline 2.50 & Qt. & \\
\hline .60 & $\begin{array}{r}10 \\
100\end{array}$ & \\
\hline $\begin{array}{r}.10 \\
.80 \\
8.00\end{array}$ & $\begin{array}{r}10 \\
100 \\
1000\end{array}$ & \\
\hline .25 & $\begin{array}{r}10 \\
100\end{array}$ & \\
\hline
\end{tabular}
$150.00 \quad 100.00 \quad 80.00$

No bulblets

$10 \quad .50$

$10 \quad .15$

$100 \quad 1.00$

$100 \quad .35$

$1000 \quad 2.50$

Each $\quad 3.00$

$100 \quad .50$

$1000 \quad 3.00$

Pkg. $\quad .10$

$1000 \quad 1.00$

$10 \quad .10$

$100 \quad 1.00$

$1000 \quad 5.00$

$10 \quad .20$

$100 \quad 1.50$

$1000 \quad 10.00$

Pkg. $\quad .10$

$1000 \quad 1.00$

$10 \quad .25$

.......

......... .........

No Bulblets

Pkg. $\quad .10$

$1000 \quad .80$

Pkg. $\quad .25$

$1000-1.60$

Each $\quad .20$

Each .25

$10 \quad 2.50$

$100 \quad 10.00$

$1000 \quad 70.00$

Pkg. .25

$1000 \quad 3.50$

Pt. $\quad 8.00$

Qt. $\quad 14.00$

Pkg. $\quad .10$

$1000 \quad 1.25$

Pt. 2.50

Qt. $\quad 4.00$

Each .50

$100 \quad 25.00$

$100 \quad 150.00$ 
$\begin{array}{llllll}\text { Per No. } 1 & \text { No. } 2 & \text { No.3 } & \text { No. } 4 & \text { No. } 5 & \text { No. } 6\end{array}$

Bulblets

Per

$\begin{array}{llllllll}\text { Mary Jane.......... } & 1 & 1.25 & 1.00 & .65 & .50 & .40 & .25\end{array}$

$10 \quad 1.00$

Mary J. Coole.......

$.25 \quad .20$

.15

$.40 \quad .25$

Pkg. $\quad .25$

Milady Imogene..... 1

2.00

1.60

1.20

.80

.60

.40

$1000 \quad 2.00$

Millionaire......... 1

Minuet...........

$\begin{array}{lll}1.50 & 1.50 & 1.00\end{array}$

1.00

.85

.75

.60

1.25

1.00

.85

10.00

8.00

6.80

100

Miss Madison. . . . . . $\quad 1 \quad .15$

……- -.......

.80

.10

.70

.60

Miss Spokane....... 1

Miss Tea Rose...... . 1

.65

.55

.45

.20

$.15 \quad .10$

1.60

Mona Lisa........ 1

.10

1.20

.80

.65

.07

3.20

2.60

.75

6.00

50.00

.50

.50

............

Each $\quad .15$

$4.50 \quad 4.00$

$42.00 \quad 35.00$

Morena............. 1

Mother Machree. .... .

Mr. Mark..........

1.00

.50

$.35 \quad .25$

.35

.25

.15

.60

$.40 \quad .30$

.45

.35

.25

$100.00 \quad 100.00 \quad 60.00$

.45

.35

.25

$\begin{array}{lll}.10 & . . . . . . & .07\end{array}$

10

Mrs. A. B. C. Dohrmann 1

Mrs. C. Edwards.....

Mrs. Dr. Norton.... 1

Mrs. E. Morrison.... 1

Mrs. F. C. Hornberger 1

10

.75
3.00

.65

.55

$\ldots . . . . .$.

$\begin{array}{lll}3.00 & 2.75 & 2.50\end{array}$

75.00

$60.00 \quad 50.00$

2.00

.10

.65

. .55

.07

.35

.25

.45

40.00

1.00

1.50

.20

.35

.25

.15

1.00

.15

.10

.35

7.50

$.70 \quad .50$

35.00

$6.00 \quad 4.00$

.40

.30

.20

$30.00 \quad 25.00$

3.00

2.00

1.50

Mrs. F. C. Peters... $\quad 1$

.10

100

1000

Mrs. H. A. Caesar... 1

4.50

45.00

.55

.07

.........

........

10.00

.25

4.00

3.50

2.40

.25

.15

2.00

.20

.-15

$\cdots . . .10$

........

.25

1.60

1.20

.80

.60

1.60

.15

.10

15.00

12.00

10.00

7.00

5.00

15

1.10

$\begin{array}{rr}\text { Mrs. J. R. Walsh.... } & 1 \\ 100\end{array}$

Mrs. L. S. Schweppe..

.15

1.10

100

100

Mrs. Leo Miller......

4.00

…… $\quad .10$

.75

5.00

4.00

3.00

.60

2.00

.80

$\cdots .-1.95$

.10

.75

.60

$\begin{array}{ll}3.00 & 2.00\end{array}$

1.00

.75

.40

100.00

Mrs. L. Douglas.....

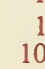

100

.15

$27.00 \quad 18.00$

9.00

6.00

$$
3.00
$$

.45

1.50

. .45

.50

4.50

.80

6.00

…... $\quad 10$

............

..................

….............

$\begin{array}{llll}5.00 & 4.00-3.00\end{array}$

$2.25 \quad 1.40$

Mrs. Kr. Prestgard. . .

1

Mrs. N. Vanderbilt... 11

Mrs. P. W. Sisson .... 1

Mrs. T. Rattray .

36.00

27.00

22.00

12.00

15

$.20 \quad .15$

.........
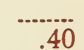

.30

$\begin{array}{llllll}.50 & .45 & .40 & .30 & .20 & .10\end{array}$

$\begin{array}{rrrrrr}4.50 & 3.50 & 3.00 & 2.00 & 1.50 & 1.00 \\ \ldots-. . . & 30.00 & 25.00 & 17.50 & 10.00 & 8.00\end{array}$

$\begin{array}{rrrrrr}4.50 & 3.50 & 3.00 & 2.00 & 1.50 & 1.00 \\ \ldots-. . & 30.00 & 25.00 & 17.50 & 10.00 & 8.00\end{array}$

$\begin{array}{lr}10 & 1.00 \\ 10 & .75\end{array}$

$10 \quad .75$

$100 \quad 6.00$

Pkg. $\quad .15$

$1000 \quad 1.00$

Pkg. $\quad .10$

$1000 \quad 1.00$

Pkg. $\quad .10$

$1000 \quad 2.00$

Pkg. $\quad .10$

$1000 \quad 1.00$

$10 \quad .50$

No Bulblets

Pkg. $\quad .10$

Each $\quad .25$

Each 5.00

Pkg. $\quad .10$

$1000 \quad 1.00$

$\begin{array}{rr}10 & .40 \\ 100 & 1.00\end{array}$

$10 \quad .50$

$100 \quad 2.00$

$1000 \quad 15.00$

Pkg. $\quad .10$

$1000 \quad 1.00$

Pt. $\quad 1.75$

Qt. $\quad 3.00$

\begin{tabular}{ll}
$10 \quad .20$ \\
\hline
\end{tabular}

$1000 \quad 3.00$

Pkg. .25

$1000 \quad 2.00$

Pkg. $\quad .15$

Pkg. $\quad .10$

$1000 \quad .50$

Qt. $\quad 1.5 C$

Each 10.06

Pkg. $\quad .25$

$1000 \quad 1.50$

Pkg. $\quad .25$

Pkg. $\quad .15$

$\begin{array}{rr}10 & .40 \\ 100 & 1.00\end{array}$

$1000 \quad 8.00$ 


\section{CHAMPLAIN VIEW GARDENS BURLINGTON, VERMONT}

\section{Collections and Combination Sets}

at Very Special Prices for March

At these special prices there can be no changes in the sets as listed and there are no further discounts except that on orders of $\$ 100$ or more from this list you can take $10 \%$ discount in cash or add $15 \%$ in bulbs. Just order by number. Collections are numbered. Prices are for cash.

\section{Everything Prepaid}

No. 1. 5 No. 1 Taro, 5 No. 3 Richard Diener, 5 No. 3 Rev. Ewbank, 5 No. 2 Scarlet Wonder, 5 No. 2 Elf, 5 No. 2 Gold, 5 No. 2 J. V. Beiren, 5 No. 2 Persia, 5 No. 2 Romance, 5 No. 2 Crimson Glow. 50 bulbs, value $\$ 2.91$ for $\$ 2.25$.

No. 2. 5 No. 2 Mrs. Schweppe, 5 No. 1 Orange Queen, 5 No. 2 Dr. Jackson, 5 No. 2 Dr. Van Fleet, 5 No. 3 E. G. Hill, 5 No. 2 Mrs. Peters, 5 No. 2 Opalescent, 5 No. 3 Quinton, 5 No. 2 Diana, 5 No. 3 Sidney Plummer. 50 bulbs, value $\$ 2.92$ for $\$ 2.25$.

No. 3. 5 No. 2 Phaenomen, 5 No. 3 Annie Laurie, 5 No. 3 Blue Torch, 5 No. 1 Marnia, 5 No. 1 Mrs. Whitcomb, 5 No. 2 Dr. Bennett, 5 No. 2 Cardinal Prince, 5 No. 3 Desdemona, 5 No. 1 Fern Kyle, 5 No. 2 St. Thomas. 50 bulbs, value $\$ 7.69$ for $\$ \mathbf{5 . 5 0}$.

No. 4. The three above collections, value $\$ 10.00$ for $\$ 9.00$.

No. 5. The following varieties in sizes from No. 5 to No. 3. Any 10 items for $\$ 2.50$. The whole set for $\$ 4.00-\mathrm{A}$. E. Kunderd, Emile Aubrun, Gold Eagle, Golden Dream, Golden Salmon, Herbstzauber, Minuet, Mrs. P. W. Sisson, Mrs, van Konynenburg, Rita Beck, Rose Mulberry, Veiled Brilliance, Mrs. Hornberger, Mrs. Ella Morrison, Golden Frills, Pfitzer's Triumph, Kunderd's Yellow Wonder.

No. 6. The following in sizes from No. 5 to No. 3. Any 5 for $\$ 5.00$. The whole list for $\$ 10.00$. Bleeding Heart, Helen Phipps, Marmora, Oraflame, Pearl of California, Ruffled Gold, Aflame, Mrs. Calvin Coolidge, Orange Wonder, Carolus Clusius, Dr. Moody, Mary Frey.

No. 7. A. E. Kunderd, 1 No. 2,2 No. 6, and 10 bts. for $\$ 1.25$.

No. 8. Aflame, 1 No. 1,1 No. 3,5 No. 6 , and 25 bts. for $\$ 6.00$. 
No. 9. Annie Laurie, 2 No. 2,12 No. 4,12 No. 5 , and 250 bts. for $\$ 5$.

No. 10. Annie Laurie, 2 No. 1, 25 No. 4, 25 No. 5 , and 500 bts. for $\$ 10$.

No. 11. Antonia Antonette, 2 No. 2, 3 No. 4, 5 No. 6, 100 bts. for $\$ 1.75$.

No. 12. Bleeding Heart, 1 No. 1,2 No. 6,5 bts. for $\$ 5.00$.

No. 13. Blue Torch, 5 No. 2, 10 No. 5,10 No. 6, 200 bts. for $\$ \mathbf{2 . 5 0}$.

No. 14. Bobby, 2 No. 2, 10 No. 6, 150 bts. for $\$ 1.50$.

No. 15. Butterboy, 50 No. 1,50 No. 3, 1 qt. bts. for $\$ 3.50$.

No. 16. Cabernet, 1 No. 1,3 No. 6,5 bts. for $\$ 3.50$.

No. 17. Cardinal Prince, 1 No. 1,1 No. 2,1 No. 3,25 No. 5,25 No. 6 , 250 bts. for $\$ \mathbf{2 . 5 0}$.

No. 18. Carolus Clusius, 1 No. 2, 5 No. 5,5 No. 6,50 bts. for $\$ 8.50$.

No. 19. Chicago, 1 No. 23 bts. for $\$ 7.00$.

No. 20. Copper Bronze, 5 No. 2, 5 No. 4, 25 No. 5, 250 bts. for $\$ \mathbf{2 . 7 5}$.

No. 21. Coryphee, I No. 2,1 No. 6,4 bts. for $\$ 8.00$.

No. 22. Dr. Moody, 1 No. 1,1 No. 4,3 No. 5,1 No. 6,10 bts. for $\$ 9.50$.

No. 23. Dr. Christ Martz, 2 No. 1, 2 No. 5,5 No. 6, 100 bts. for $\$ .75$.

No. 24. Dr. W. Van Fleet, 100 No. 4, 200 No. 5, 200 No. 6, 1000 bts. for $\$ 4.00$.

No. 25. E. B. Williamson, 10 No. 3, 25 No. 6,1000 bts. for $\$ 1.50$.

No. 26. Elf, 5 No. 3, 100 No. 5, 100 No. 6, 1000 bts. for $\$ 1.75$.

No. 27. Emile Aubrun, 1 No. 2, 1 No. 3, 10 No. 6, 100 bts. for $\$ 2.00$.

No. 28. Emile Aubrun, 4 No. 2, 2 No. 3, 25 No. 5,25 No. 6, 500 bts. for $\$ 6.00$.

No. 29. Emile Aubrun, 25 No. 2, 250 No. 5, 50 No. 6, 1 qt. bts. for $\$ 47.50$.

No. 30. Flaming Sword, 5 No. 2, 25 No. 4, 100 No. 5, 100 No. 6, 100 bts. for $\$ 5.00$

No. 31. Gertrude Errey, 1 No. 3, 6 No. 6, 100 bts. for 50 cents.

No. 32. Gertrude Pfitzer, 1 No. 2,1 No. 5,5 bts. for $\$ 6.00$.

No. 33. Gloriana, 1 No. 1, 1 No. 3, 10, No. 5, 25 No. 6, 250 bts. for $\$ 3.50$.

No. 34. Gloriana, 1 No. 2, 10 No. 5, 100 bts. for $\$ \mathbf{2 . 0 0}$.

No. 35. Gold Eagle, 1 No. 2, 5 No. 4, 50 bts. for $\$ \mathbf{2 . 0 0}$.

No. 36. Golden Dream, 1 No. 1, 1 No. 2, 5 No. 4,10 No. 5, 25 No. 6, 250 bts. for $\$ 12.00$.

No. 37. Golden Dream, 1 No. 2, 5 No. 5, 100 bts. for $\$ 3.75$.

No. 38. Golden Frills, 1 No. 2, 2 No. 4,10 No. 6, 100 bts. for $\$ 1.50$.

No. 39. Golden Frills, 1 No. 1, 1 No. 2, 5 No. 3, 25 No. 4, 100 No. 5 , 100 No. 6, 1000 bts. for $\$ 17.50$.

No. 40. Golden Salmon, 1 No. 2, 4 No. 5, 5 No. 6, 100 bts. for $\$ 2.00$.

No. 41. Goldielocks, 1 No. 1,5 bts. for 60 cents.

No. 42. Harbinger, 1 No. 2,2 No. 4,5 No. 6,25 bts. for $\$ \mathbf{1 . 2 5}$.

No. 43. Heavenly Blue, 1 No. 4, 1 No. 6,3 bts. for $\$ \mathbf{5 . 5 0}$.

No. 44. Heinrich Kantzleiter, 1 No. 1, 1 No. 3, 10 No. 5, 10 No. 6, 100 bts. for $\$ 2.00$.

No. 45. Helen Phipps, 1 No. 2, 5 No. 6,10 bts. for $\$ 6.00$.

No. 46. Herbstzauber, 2 No. 3,10 No. 5,10 No. 6,100 bts. for $\$ 3.25$.

No. 47. High Nye, 1 No. 2, 3 No. 4, 5 No. 5,100 bts. for $\$ 1.50$.

No. 48. I wa, 3 No. 3,10 No. 6,100 bts. for $\$ 1.00$. 
No. 49. King Tut, 1 No. 1,1 No. 6,25 bts. for $\$ 4.00$.

No. 50. Kundred's Apple Blossom, 2 No. 2, 5 No. 3, 100 No. 5, 100 No. 6, 1000 bts. for $\$ 4.50$.

No. 51. Kunderd's Yellow Wonder, 1 No. 2, 2 No. 4, 10 No. 5, 10 No. 6 , 100 bts. for $\$ \mathbf{2 . 5 0}$.

No. 52. Lilac Wonder, 1 No. 3, 3 No. 5, 100 bts. for 50 cents.

No. 53. Lorice, 1 No. 2, 2 No. 3, 5 No. 5, 5 No. 6, 100 bts. for $\$ 2.00$.

No. 54. Loyalty, 3 No. 1 for $\$ 5.00$.

No. 55. Mammoth White, 1 No. 1, 1 No. 5, for $\$ 10.00$

No. 56. Marmora, 1 No. 2, 1 No. 5, 2 No. 6, 10 bts. for $\$ 5.00$.

No. 57. Marmora, 1 No. 1,10 bts. for $\$ 3.00$.

No. 58. Mlarmora, 1 No. 1, 3 No. 5, 5 No. 6, 25 bts. for $\$ 10.00$.

No. 59. Marnia, 2 No. 1, 2 No. 3, 25 No. 5, 100 bts. for $\$ 1.00$.

No. 60. Marnia, 5 No. 1, 10 No. 3, 100 No. 5, 1000 bts. for $\$ 5.00$.

No. 61. Marnia, 25 No. 1, 25 No. 4, 250 No. 5, 500 No. 6, 1 quart bts. for $\$ 25.00$.

No. 62. Marshall Foch, 25 No. 3, 100 No. 5, 200 No. 6, 1000 bts. for $\$ 3.50$.

No. 63. Mary Frey, 1 No. 1, 1 No. 4, 2 No. 5, 20 bts. for $\$ 9.00$.

No. 64. Minuet, 1 No. 3, 2 No. 5, 3 No. 6, 10 bts. for $\$ 2.00$.

No. 65. Miss Tea Rose, 2 No. 2, 5 No. 5, 100 bts. for 50 cents

No. 66. Mrs. Calvin Coolidge, 1 No. 3, 5 bts. for $\$ 2.00$.

No. 67. Mrs. F. C. Hornberger, 1 No. 2, 2 No. 4, 5 No. 5, 100 bts. for $\$ 3.25$.

No. 68. Mrs. F. C. Hornberger, 2 No. 2, 5 No. 4, 25 No. 5,25 No. 6 , 500 bts. for $\$ 10.75$.

No. 69. Mrs. H. A. Caeser, 2 No. 3, 5 No. 6, 100 bts. for 55 cents.

No. 70. Mrs. H. W. Whitcomb, 2 No. 2, 5 No. 3, 10 No. 4, 250 bts. for $\$ 1.50$.

No. 71. Mrs. L. S. Schweppe, 10 No. 2, 25 No. 3, 100 No. 4, 100 No. 5 , 250 No. 6,1 qt. bts. for $\$ 5.00$.

No. 72. Mrs. P. W. Sisson, 1 No. 3, 3 No. 5, 10 No. 6, 100 bts. for $\$ 1.75$.

No. 73. Mrs. P. W. Sisson, 2 No. 2, 2 No. 3, 5 No. 5,50 No. 6, 400 bts. for $\$ 7.50$.

No. 74. Mrs. Konynenburg, 1 No. 2, 5 No. 6, 10 bts. for $\$ 2.50$.

No. 75. Mrs. Konynenburg, 1 No. 2, 10 No. 5, 25 No. 6, 100 bts. for $\$ 15$

No. 76. Olive Goodrich, 1 No. 1, 5 No. 5, 100 bts. for $\$ 2.00$.

No. 77. Opalescent, 10 No. 2, 100 No. 4, 250 No. 5, 500 No. 6, 1 quart bts. for $\$ 8.75$.

No. 78. Oraflame, 1 No. 1,1 No. 4,1 No. 6,10 bts. for $\$ 5.50$.

No. 79. Orange Queen, 5 No. 1, 100 No. 4, 250 No. 5, 250 No. 6, 1 qt. bts. for $\$ 5.50$.

No. 80. Orange Flame, 2 No. 2, 5 No. 3, 25 No. 6, 250 bts. for $\$ 1.50$.

No. 81. Orange Wonder, 1 No. 1, 1 No. 5, 1 No. 6, 10 bts. for $\$ 5.50$.

No. 82. Orchid Lady, 1 No. 4,5 bts. for $\$ 1.00$

No. 83. Patricia Carter, 1 No. 3, 10 bts. for $\$ 2.00$.

No. 84. Pauline Kunderd, 1 No. 2, 1 No. 6, 2 bts. for $\$ 5.00$.

No. 85. Paul Pfitzer, 1 No. 2, 2 No. 4 , $;$ No. 6, 100 bts. for $\$ 5.00$.

No. 86. Pearl of California, 1 No. 2, 2 No. 4,10 bts. for $\$ 4.00$. 
No. 87. Persia, 10 No. 1,100 No. 5,250 No. 6,1 qt. bts. for $\$ 5.50$.

No. 88. Phaenomen, 5 No. 2, 100 No. 4, 250 No. 5, 250 No. 6, 1000 bts. for $\$ 7.50$.

No. 89. Pride of Portland, 1 No. 2, 2 No. 5,3 bts. for $\$ 6.00$.

No. 90. Purest of All, 2 No. 2, 5 No. 3, 25 No. 5,25 No. 6,500 bts. for $\$ 4.00$

No. 91. Queen of Orange, 1 No. 1, 5 No. 4, 10 bts. for $\$ 2.00$.

No. 92. Quinton, 25 No. 2, 100 No. 3, 200 No. 4,500 No. 5,500 No. 6, 1 qt. bts. for $\$ 12.00$.

No. 93. Red Tornado, 1 No. 2,1 No. 5,5 bts. for $\$ 8.00$.

No. 94. Rita Beck, 1 No. 1,3 No. 5,5 No. 6,10 bts. for $\$ 3.50$.

No. 95. Rita Beck, 1 No. 1,3 No. 3,10 No. 4,15 No. 5,25 No. 6 , 250 bts. for $\$ 7.50$.

No. 96. Rose Mist, 2 No. 1, 2 No. 3, 5 No. 5,50 bts. for $\$ 1.75$.

No. 97. Ruffled Gold, 1 No. 3,1 No. 5,10 bts. for $\$ 7.50$.

No. 98. Sacajawea, 1 No. 2, 3 No. 6, 100 bts. for 50 cents.

No. 99. St. Thomas, 10 No. 3, 100 No. 5, 200 No. 6, 500 bts. for $\$ 2.50$.

No. 100. St. Thomas, 5 No. 2,10 No. 3,25 No. 4,100 No. 5,100 No. 6 , 1000 bts. for $\$ 5.00$.

No. 101. St. Thomas, 10 No. 2, 10 No. 3, 250 No. 5, 250 No. 6, 1 qt. bts. for $\$ 9.50$.

No. 102. Sidney Plummer, 5000 No. 6, for $\$ 22.50$.

No. 103. Sidney Plummer, 1000 No. 5, 2000 No. 6, 2 qts. bts. for $\$ 18$.

No. 104. Sidney Plummer, 1000 No. 7 , for $\$ 3.00$.

No. 105. Sidney Plummer, 250 No. 5, 500 No. 6, 1 qt. bts. for $\$ 5.00$.

No. 106. Star of the Sea, 1. No. 2, 5 bts. for $\$ 2.50$.

No. 107. Taro, 10 No. 1, 100 No. 3, 250 No. 5, 500 No. 6, 1 qt. bts. for $\$ 10.50$.

No. 108. Taro, 10 No. 1,50 No. 3,250 No. 5, 250 No. 6,1 pt. bts. for $\$ 6$.

No. 109. Taro, 1000 No. 7 for $\$ 5.00$.

No. 110. Tobersun, 3 for $\$ 5.00$.

No. 111. Tycko Zang, 10 No. 2,10 No. 3, 100 No. 6, 1000 bts. for $\$ 4.00$.

No. 112. Veilchenblau, 3 No. 2, for $\$ 1.35$.

No. 113. W. C. Badger, 2 No. 2, 100 No. 6,100 No. 7, 500 bts. for $\$ 2.00$.

No. 114. Collection of 100 bulbs 1 inch and up in diameter in at least 30 varieties and in many different shades for $\$ 3.00,50$ for $\$ 1.75$, 25 for $\$ 1.00$. These are all standard varieties and most of them are listed in my catalog. If the varieties were kept separate and labeled they would be worth at least twice the price charged. I believe this to be the finest collection offered by any grower at the price, and better than most collections regardless of price. I have many fine testimonials from people who had this collection last year.

No. 115. Collection of Prims. 100 one inch up in size, 25 or more kinds, same price as above collection. 50 and 25 same as above.

No. 116. Collection same as No. 114 but in size from $1 / 2$ inch to 1 inch. These will practically all bloom but the flowers will not be so large the first year as the larger bulbs will produce. They will bloom later. 100 for $\$ 1.50$. 
Mrs. R. Lohrman... $\quad 1$

Mrs. R. Cunningham . 1

Mrs. S. Arnold...... 1

Mrs. V. Konynenburg.

Mrs. W. E. Clark... . 1

Mrs. W. Richardson. . 1

Nancy Hanks........ . 1

Nectarine

Norma Talmadge.... 1

Olive Goodrich....... 1

Opalescent ........

Oraflame.......... 1000

10

Orchid Lady......... 1

Orange Flame......

Orange Glow ........ 1

Orange Wonder...... 1

Osalin............ 1

Pasadena.......... 1

Pauline Kunderd..... 1

Paul Pfitzer......... 1

Pearl of California... 1

Persia

Phaenomen
Per

100

10

100

10

100

1000

10

10

100

10

100

10

100

10

100

10

100

10
100

No. $1 \quad$ No. $2 \quad$ No. 3

3.00

4.00

12.00

10

.80

.30

1.50

10.00

6.00

3.50

5.00

4.00

33.50

1.00

2.00

.10

4.00

33.50

1.20

5.00

6.50

60.00

$2.00 \quad 1.75$

8.00

80.00

6.00

1000

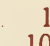

100

1000

12.50 $\begin{array}{lrr}. .3 . . . & 1.75 & 1.60\end{array}$
.10

..........

$\begin{array}{ll}.75 & .65 \\ .50 & 40\end{array}$

2.25

.50

.40

1.50

3.20

1.00

9.00 100.00

.10

.75

.10

.10
.75

.50

.40

.10

.65

.25

.35

12.00

10.00

.15

1.00

.10

$4.00 \quad 3.00$

$\begin{array}{lll}.80 & .70 & .55\end{array}$

5.50

7.00

70.00

.10

80

.07

.55

1.50

.30

2.40

7.00

80.00

.07

4.00

.07

.60

3.00

.20

1.00

4.80

.07

4.00

.30

.25

2.00

.07

3.00

2.50

20.00

.75

.15

1.20

6.00

.55

2.50

20.00

2.00

15.00

.20

1.50

8.00

.70

2.50

.45

35.00

3.50

30.00

$1.50 \quad 1.35$

$6.00 \quad 5.00$

60.00

45.00

.07

.70

5.00

45.00

.60

4.00

36.00

3.00

27.00

.25

.20
1.25

.15

.10
.80

6.00

54.00

90.00
No. 5 No. 6

.25

.15

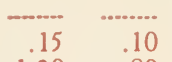

$1.20 \quad .80$

$.60 \quad .40$

$5.00 \quad 3.50$

$45.00 \quad 30.00$

$2.20 \quad 1.40$

.40

1.50

.30

1.00

7.00

.10

.50

2.00

.40

1.00

.35

.25

2.00

.15

.15

1.00

1.00

.10

.20

.10

1.60

12.00

1.50

6.80

1.00

12.00

9.00

.50

.40

.10

3.80

.60

2.00

.35

.25

1.50

1.00

12.00

.15

1.25

6.00

9.00

.10

1.00

4.00

$.60 \quad .50$

.35

2.50

22.00

1.25

4.00

36.00

.25

2.00

15.00

1.00

3.00

27.00
$.40 \quad \cdots 35$
$.40 \quad .30$

$2.00 \quad 1.00$

$\begin{array}{rr}.70 & .50\end{array}$

$4.50 \quad 3.00$

$40.00 \quad 27.00$
Bulalets

Per

Pkg. $\quad .10$

$1000 \quad .90$

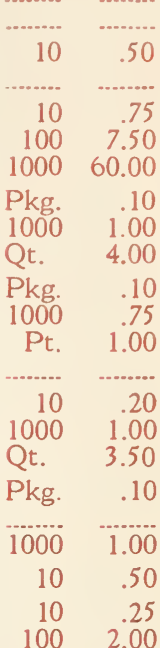

Pkg. $\quad .10$

$1000 \quad 1.00$

Pt. $\quad 2.00$
Pt.

$10 \quad 3.00$

$100 \quad 20.00$

Each .15

Pkg. $\quad .15$

$1000 \quad 2.50$

Pkg. $\quad .10$

$10 \quad 3.00$

$100 \quad 20.00$

$10 \quad .15$

$100 \quad .60$

$1000 \quad 5.00$

Pkg. $\quad .10$

$100 \quad .50$ 
$\begin{array}{llllll}\text { Per No.1 No. } 2 & \text { No. } 3 & \text { No. } 4 & \text { No. } 5 & \text { No. } 6\end{array}$

Bulblets

Per

Pink Cloud..........

.25

$.20 \quad .15$

.10

Pink Lily ......... $\begin{array}{r}1 \\ 10 \\ 100\end{array}$

.10

Pink Wonder........

.70

.60

.07

35

-.........

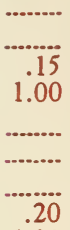

$10 \quad .20$

Pkg. $\quad .10$

$1000 \quad 1.00$

$\begin{array}{llllll}4.00 & 3.00 & 2.00 & 1.00 & \text { Qt. } & 4.00\end{array}$

.10

.60

Pola Negri .........

100

President Harding.... 1

Pride of Oregon.......

Pride of Portland....

Primrose Princess....

Princely ...........

Prince of Wales...... 1

Purest of All .......

10

Purple Glory . ............

.10
.80

$\begin{array}{lr} & 10 \\ & 100 \\ \text { Purple Queen........ } & 1 \\ 10\end{array}$

Queen Mary........

Quinton............

100

1000

Ravenna Cream.....

1

Reah............

1

Red Fire.........

100

Red Tornado.........

.40

3.50

...... .07

.10

.25

8.00

5.00

1.00

.50

.40

.

3.00

.40

1.15

.10

$\begin{array}{rr}\cdots . . . . & \cdots . . . . \\ .30 & .20 \\ 1.50 & 1.00\end{array}$

Pkg. $\quad .10$

.20

1.50

1.25

1.00

.75

3.00

3.50

2.75

2.00

1.00

.80

.60

.50

.40

.25
1.00

$20 \quad 15 \quad 10$

2.00

1.50

Pkg. $\quad .10$

$1000 \quad 75$

Qt. $\quad 2.50$

$10 \quad .20$

$100 \quad 1.50$

Each $\quad .40$

Each .35

$\begin{array}{ll}10 & .50\end{array}$

$100 \quad 2.50$

$\begin{array}{lll}.10 & . . . . . . . . . & .07\end{array}$

.60

.50

.40

.30

...........

...................

$.30 \quad .25$

.20

.15

.10

2.50

2.00

1.60

1.20

.10

.80

07

10.00

.

.........

.65

.55

.45

.35

.25

$4.00 \quad 3.00$

2.20

.35
3.00

.30

.25

.20
1.50

.15

1.50

1.25

1.00
4.00

2.00

.50

1.00

6.00

4.00
.07

3.00

2.50

2.00

.10
$\ldots . . .$.

-.......

3.20

.40

.25

.15

.10

22.50

17.00

11.25

1.50

.35

.30

.25

…….

.15

6.50

2.00

1.50

1.25

1.00

.07
.55

.45

.75

.65

3.00

2.50

9.00

8.00

6.85

5.50

$.65 \quad .55$

Renown............

Rev. Ewbank........

100

Rich. Diener........

1
10
100

$\begin{array}{cr}.1 . & .07 \\ -.55 & 45\end{array}$

4.00

3.20

2.5

.10

.65

.55

.07

10
100

1000

Rippling Waters.....

45.00

4.00

35.00

5.00

4.50

3.20

.35

.7

.10

.30

$\ldots . . . .$.

1.80

1.00

4.25

3.00

$2.00 \quad 1.50$

$3.75 \quad 3.00$

$25 \quad-20$

Rita Beck..........

15.00

12.00

$1.00 \quad .85$

$8.00 \quad 6.75$

1.80

.25

1.00

.20

$2.00 \quad 1.00$

$15.00 \quad 8.00$

R. J. Kunderd. ......

120.00

90.00

$75.00 \quad 60.00$

.75

$5.50 \quad 4.50$

$45.00 \quad 35.00$

1
10
100

.15

.80

.10

.65

3.50

$.40 \quad .25$

Roderick Dhu......

3.00

2.50

2.00

2.25

1.40

Romance..........

.10

.07

.60
4.00

.50

.40

3.00
27.00

2.25

.30

.20

...........

10

.20

Pkg. $\quad .10$

$10 \quad .10$

$100 \quad .50$
$000 \quad 2.50$

Pkg. $\quad .10$

$\begin{array}{rr}10 & .30 \\ 1000 & 2.00\end{array}$

$10 \quad .65$

$100 \quad 2.00$

Pkg. $\quad .10$

$1000 \quad .90$

Pt. $\quad 1.50$

Qt. $\quad 2.50$

$10 \quad .15$

$100 \quad 1.00$

Pkg. $\quad .10$

$1000 \quad 1.25$

Qt. $\quad 3.00$

Each $\quad .60$

Pkg. $\quad .10$

$1000 \quad 1.00$

Pkg. $\quad .10$

$1000 \quad 1.00$

Qt. $\quad 6.00$

Each $\quad .40$

$10 \quad .80$

$100 \quad 7.00$

$1000 \quad 60.00$

Pkg. $\quad .25$

$1000 \quad 1.00$

13.50

8.00

.10

4.50

\begin{tabular}{rr}
$\cdots$ & 100 \\
\hline Pkg. & .10 \\
1000 & .75 \\
Qt. & 2.00
\end{tabular} 


\section{$\begin{array}{llllll}\text { Per No.1 No. } 2 & \text { No.3 } & \text { No. } 4 & \text { No. } 5 & \text { No. } 6\end{array}$}

Rose Ash.........

Rose Mulberry. .

10

.10

.65

...........

1.00

.75

.07

.45

.........

..........

3.00

Ruffled Gold.....

10.00

3.00

.50

.40

.35

.15

3.00 $50.00 \quad 50.00$

5.00

5.00

2.50

25.00

25.00

15.00

2.50

200.00

Ruth Huntington....<smiles></smiles>

Sacajaweв

10

10

St. Thomas.........

1000

San Mateo......... 1

Saraband

10

100

Scarlet Wonder...... 1

1000

Sheila.

80

.50

4.00

.65

.07

35

.25

.25

2.00

8.00

70.00

2.80

2.00

.20

1.50

6.50

60.00

.15

1.20

5.00

45.00

.70

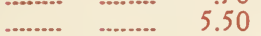

.30

2.40

.25

2.00

$16.00 \quad 14.00$

.45
.20
1.50
.10
.80
4.00

36.00

.60

4.50

.20

1.60

12.50

.10

.75

4.00

.07

.65
4.00

32.00

Sidney Plummer.....

Smoke.

Star of the Sea....... 1

Sulphur Frills....... 1

Sweet Rose........ 1

100

Sympathy.

Taro.

$$
\begin{array}{r}
1 \\
10 \\
100
\end{array}
$$

1

10

100

Thais Valdemar..... 1

The President........

Titan............. I

Thistle...........

Thos. T. Kent...... 1

Thos. A. Edison..... 1

Tobersun.........

Trosseau Pink...... 1

.10

$1.00 \quad .80$

1.25

2.00

.30

2.50

2.00

10.00

8.00

.25

1.50

6.00

.60

.10

.65

.07
.55

.20

1.25

.45

.10

.80

.07

.70

.60

.50

3.00

2.00

40.00

32.00

25.00

18.00

.35

.30

.25

.20

$2.50 \quad 2.00$

1.60

1.20

$.20 \quad .15$

.10

.50

.40

.30

.20

.10

.65

.10

.85

.75

$8.00 \quad 8.00$

2.00

2.00

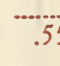

Sunkist Orange......

BL LBLETS

Per

Pkg. $\quad 10$

$10 \quad .50$

No Bulblets

Each $\quad .50$

$10 \quad 2.50$

$100 \quad 25.00$

Pkg. $\quad .10$

$1000 \quad 1.00$

Pkg. $\quad .15$

$1000 \quad 2.50$

Pkg. $\quad .10$

$1000 \quad 2.00$

Qt. $\quad 3.00$

$15 \quad 1.00$

$100 \quad 2.50$

$10 \quad .25$

$100 \quad 1.00$

$1000 \quad 5.00$

Pkg. $\quad .10$

$1000 \quad .50$

Qt. $\quad 2.50$

Pkg. $\quad 18.00$

Pkg. $\quad .10$

$1000 \quad 1.50$

Pkg. $\quad .10$

$1000 \quad 1.00$

Qt. $\quad 2.50$

Pk. $\quad 15.00$

Pkg. $\quad .10$

$1000 \quad 1.00$

Qt. $\quad 4.00$

$10 \quad 2.00$

.....

.15

1.00

$\begin{array}{lll}5.00 & 3.50 & 2.50\end{array}$

.10

.35

.25

$\begin{array}{lll}3.00 & 2.00 \quad 1.00\end{array}$

$.40 \quad .30$

1.60

14.00

1.25

.15

.10

.80

.60

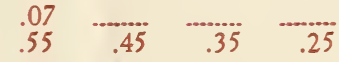

.07

.65
No Bulblets

$10 \quad .25$
100

$100 \quad 1.50$

$1000 \quad 4.00$

Pkg. $\quad .10$

$1000 \quad 1.00$

Pkg. $\quad .10$

$1000 \quad 1.00$

Pt. $\quad 1.50$

Qt. $\quad 2.50$

Pkg. $\quad .10$

$1000 \quad 2.00$

Pkg. $\quad .10$

$10 \quad .35$

Pkg. $\quad .10$

Pkg. $\quad .10$

$1000 \quad 1.00$

Each $\quad .30$

No Bulblets 
$\begin{array}{llllll}\text { Per No.1 No. } 2 & \text { No.3 } & \text { No. } 4 & \text { No. } 5 & \text { No. } 6\end{array}$

Bulblets

\subsection{0}

Twilight

Twin Fires..........

Tycko Zang......... 1

100
1000

Tyrian Beauty....... 10

Uhlan Chief........ 1

Veilchenblau........

Veiled Brilliance..... 100

Virginia............

100

Virginia Hale........

Waban........... 1

Waikiki........... 1

Watermelon......... 1

Wedgewood..........

W. H. Phipps........

Wm. G. Badger......

100

W. J. Bryan..........

Wm. Kent..........

Wm. McGavin.......

*One No. 5 or 6 free with each large bulb.

Per

Pkg. $\quad .10$

$\begin{array}{rr}\cdots \\ \mathrm{Pkg} . & .25 \\ 1000 & 1.75\end{array}$

$\begin{array}{cr}1000 & 1.75 \\ \text { Qt. } & 7.00\end{array}$

Pkg. $\quad .10$

$100 \quad .40$

Pkg. $\quad .10$

$1000 \quad, 1.50$

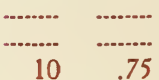

Pkg. $\quad .10$

$1000 \quad 1.50$

Qt. $\quad 2.50$

Pkg. $\quad .10$

$1000 \quad 1.00$

$\begin{array}{ll}10 & .20\end{array}$

Pkg. $\quad .10$

$1000 \quad 1.00$

Pkg. $\quad .10$

Each .15

$10 \quad .50$

Pkg. $\quad .10$

$1000 \quad .90$

Qt. $\quad 5.00$

No Bulblets

No Bulblets $10 \quad .35$

\section{PRIMULINUS}

Ada De Poy........ $\quad 1$

Aflame.

Alice Tiplady.......

Alma Gluck.........

10
100
1000

Apricot Glow........

$$
\begin{array}{r}
1 \\
100
\end{array}
$$

Athene........... 1

Baby Mine......... 1

Buddy Worden......

Butterboy...........

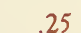

.25
1.25

$3.00-2.50$

$\begin{array}{lll}25.00 & 20.00 \quad 16.00\end{array}$

150.00125 .00

90.00

$\begin{array}{lll}.10 & -.-1.07 & .07\end{array}$

$\begin{array}{llll}.65 & -. .55 & .07\end{array}$

$\begin{array}{lll}.10 & \ldots . . . . . . . & .07\end{array}$

$\begin{array}{lll}.60 & .50 & .40\end{array}$

$$
\begin{array}{rrr}
5.00 \quad 4.00 \quad 3.00
\end{array}
$$

-... 25.00

$2.00 \quad 1.75$

$\begin{array}{lll}16.00 & 1.700 \quad 1.50\end{array}$

$150.00 \quad 130.00 \quad 110.00$

.15

.50

$\ldots . .$.
$\cdots . .$.

$.40 \quad .30$

.35

2.50

.10

3.50

25.00
$.27 \quad .20$

1.50

$\begin{array}{rr}.60 & .07 \\ 3.00 & .50\end{array}$

$3.00 \quad 2.50$

$20.00 \quad 16.00$
.75

1.50

12.00

70.00

.

.30

2.00
16.00

1.25

10.00

90.00$$
.25
$$

1.25

.40

2.00

12.00
.60

1.00

8.00

50.00

40.00

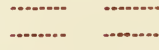

.25

1.00

8.00

1.00

7.00

60.00

.20

.75
6.00

.75

5.00

40.00

.20

-....-..-

.80

$\begin{array}{cc}.30 & .20\end{array}$

$.00 \quad .50$
$10 \quad .20$

$100 \quad 1.50$

$10 \quad 1.25$

$100 \quad 5.00$

$1000 \quad 40.00$

Pkg. $\quad .10$

Pkg. $\quad .10$

$1000 \quad .50$

Pr. $\quad 1.00$

Qt. $\quad 2.00$

$10 \quad 1.00$

$100 \quad 7.00$

$1000 \quad 50.00$

Pkg. $\quad .10$

$\begin{array}{ll}10 & .25\end{array}$

$10 \quad .25$

Pkg. $\quad .10$

$1000 \quad .75$

Pt. $\quad 1.25$ 
Per No.1 No. 2 No.3 No.4 No.5 No. 6

Cara Mia ..........

Copper Bronze ......

Copper Gold . . . . . . .

Cygnus...............

E. B. Williamson

Enchantress.........

Ethelyn.

Firy Knight.........

Golden Frills

1000

Golden Gleam....... 1

Golden Orange........

Golden Tinge........

Goldielocks..........

Harbinger.......... 1

Improved Tiplady....

Jap.

King of Oranges.....

Leen Wone.

Lilac Old Rose.......

Marigold.

Mrs. Cal. Coolidge. .

Oneta. .

Orange Queen.......

Pansy............ 1

Patricia Carter......

Queen of Orange.....

Rose Mist.......... 1

Salmon Beauty..... 100

Salmon Buff
100

1000 1

10

5.00

45.00

.30

2.50

15.00

6.00

15

10

.60

4.00

.25

.25

1.25

8.00

.25

.50

32.00

32.00

10

.10

1.00

.10

.60

.50

.50

4.00

.50

$$
\ldots
$$

.50

.40

.25

2.00

.15

1.20

.50

3.00

1.00

1

10

1

1

1

1

$\begin{array}{rr}20.00 & 16.00 \\ & .80\end{array}$

$+.00 \quad 3.00$

30.00

.15

1.20

1.80

1.00

.35

3.00

20.00

.10
.65

3.50

1000

…... 2.50

20.00

.25

2.00

.20

1.50

8.00

4.00

$\begin{array}{r}5.00 \\ -10 \\ \hline\end{array}$

….. $\quad .07$

3.50

32.00

.20

.20

1.00
7.00

.20

.45

$27.50 \quad 20.00$

150.00

3.00
24.00

.15

.15

6.00

.15

.35

3.00

.70

.07
.60

.60

.07

$.40 \quad .30$

.40

.30

2.40
.35

1.50

12.50
.25

.35

.30

.15

1.60

1.20

.10

1.00

.40

.80

.30

.60

.60

2.50
16.00

24.00

.10

1.00

.80

$1.60 \quad 1.40$

.60

$.30 \quad .25$

$2.50 \quad 2.00$

16.00

12.00

.55

3.00

.07
.45
.5

2.50

16.00

.10
.60

3.50
10
80

15.00

1.50

15

.10

1.20

6.00
50.00

100

4.50
40.00

.

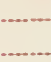

40.00

.30

16.00

.10

.10

.65

4.00

.10

.25

2.00

120.00

.50

.40

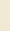

.50

.07
.40

2.50
Bulblets

$\mathrm{Per}$

Each $\quad .40$

$100 \quad 20.00$

$10 \quad .25$

$100 \quad 1.50$

$1000 \quad 2.00$

Qt. $\quad 6.00$

$10 \quad .15$

Pkg. $\quad .10$

$1000 \quad 1.50$

Qt. $\quad 3.00$

$8.00 \quad 5.00$

$10 \quad .25$

$10 \quad .25$

$1000 \quad 2.50$

Qt. $\quad 9.00$

$10 \quad .25$

$10 \quad .20$

$1000 \quad 4.00$

Qt. 15.00

Pkg. $\quad .10$

$10 \quad 1.00$

Pkg. $\quad .10$

$10 \quad .50$

$10 \quad .20$

$100 \quad 1.50$

$10 \quad .25$

$100 \quad 1.00$

$1000 \quad 7.50$

$10 \quad .40$

$10 \quad .50$

$10 \quad .25$

Pkg. $\quad .10$

$10 \quad .35$

Each .30

\begin{tabular}{ll}
$10 \quad 2.00$ \\
\hline
\end{tabular}

Each $\quad .20$

Pkg. .10

$1000 \quad .80$

Qt. 2.50

$\begin{array}{lll}2.00 & 1.75 & 1.00\end{array}$

Pkg. $\quad 15$

$10 \quad .75$

$10 \quad 1.00$

$10 \quad .20$

$100 \quad 1.50$

$1000 \quad 12.00$

Pkg. $\quad 10$

$1000 \quad .75$

$\begin{array}{ll}\text { Qt. } & 1.50 \\ \text { Pkg. } & 10\end{array}$

$1000 \quad .75$ 


\begin{tabular}{|c|c|c|c|c|c|c|c|c|c|}
\hline & Per & No. 1 & No. 2 & No. 3 & No. 4 & No. 5 & No. 6 & $\begin{array}{l}\text { BulbLr } \\
\text { Per }\end{array}$ & \\
\hline Salmon Glow. & $\begin{array}{r}1 \\
10 \\
100\end{array}$ & $\begin{array}{r}.25 \\
2.00 \\
8.00\end{array}$ & $\begin{array}{r}.20 \\
1.60 \\
7.50\end{array}$ & $\begin{array}{r}.15 \\
1.20 \\
5.60\end{array}$ & $\begin{array}{r}.10 \\
.80 \\
4.00\end{array}$ & $\begin{array}{r}.60 \\
2.50\end{array}$ & $\begin{array}{r}.40 \\
2.00\end{array}$ & $\begin{array}{r}10 \\
1000 \\
\text { Qt. }\end{array}$ & $\begin{array}{r}.10 \\
2.00 \\
4.00\end{array}$ \\
\hline Scarlet Bedder..... . . & 1 & .75 & .65 & .50 & .40 & .30 & ......... & 10 & .50 \\
\hline Shell Pink. . . . . . . . & $\begin{array}{r}1 \\
10 \\
100\end{array}$ & $\begin{array}{r}.10 \\
.80 \\
4.00\end{array}$ & $\begin{array}{r}.70 \\
3.00\end{array}$ & $\begin{array}{r}.07 \\
.60 \\
2.50\end{array}$ & $\begin{array}{r}.50 \\
2.00\end{array}$ & $\begin{array}{r}.40 \\
1.00\end{array}$ & $\begin{array}{l}.30 \\
.60\end{array}$ & $\begin{array}{c}\text { Pkg. } \\
1000 \\
\text { Qt. }\end{array}$ & $\begin{array}{r}.10 \\
.80 \\
2.00\end{array}$ \\
\hline Sunset Glow........ & $\begin{array}{r}1 \\
10\end{array}$ & $\begin{array}{l}.10 \\
.80\end{array}$ & .70 & $\begin{array}{l}.07 \\
.60\end{array}$ & .50 & .40 & .30 & $\begin{array}{l}\text { Pkg. } \\
1000\end{array}$ & $\begin{array}{r}.10 \\
1.00\end{array}$ \\
\hline Sweeter Seventeen.... & 1 & .50 & .40 & .30 & .25 & .20 & .15 & 10 & .40 \\
\hline Taurus............ & $\begin{array}{r}1 \\
10\end{array}$ & .10 & .70 & $\begin{array}{l}.07 \\
.60\end{array}$ & .50 & .40 & .30 & $\begin{array}{l}\text { Pkg. } \\
1000\end{array}$ & $\begin{array}{r}.10 \\
1.50\end{array}$ \\
\hline Temblor. & $\begin{array}{r}1 \\
10\end{array}$ & $\begin{array}{r}.25 \\
2.00\end{array}$ & $\begin{array}{r}.20 \\
1.60\end{array}$ & $\begin{array}{r}.15 \\
1.20\end{array}$ & $\begin{array}{l}.10 \\
.80\end{array}$ & .60 & .40 & 10 & .20 \\
\hline The Orchid.......... & 1 & 1.00 & .80 & .60 & .40 & .30 & .20 & 10 & .50 \\
\hline Today...... & $\begin{array}{r}1 \\
10\end{array}$ & $\begin{array}{r}.20 \\
1.25\end{array}$ & $\begin{array}{l}.15 \\
.90\end{array}$ & $\begin{array}{l}.10 \\
.70\end{array}$ & .50 & .40 & .25 & $\begin{array}{l}\text { Pkg. } \\
1000\end{array}$ & $\begin{array}{l}.15 \\
.75\end{array}$ \\
\hline Virginia Lou. . & $\begin{array}{r}1 \\
10\end{array}$ & $\begin{array}{r}.20 \\
1.20\end{array}$ & $\begin{array}{l}.15 \\
.90\end{array}$ & $\begin{array}{l}.10 \\
.70\end{array}$ & .50 & .40 & .25 & Pkg. & .15 \\
\hline Yellow Treasure..... & $\begin{array}{r}1 \\
10 \\
100\end{array}$ & $\begin{array}{r}.10 \\
.60 \\
3.00\end{array}$ & $\begin{array}{r}.50 \\
2.00\end{array}$ & $\begin{array}{r}.07 \\
.40 \\
1.25\end{array}$ & $\begin{array}{l}.30 \\
.75\end{array}$ & …..... & $\begin{array}{c}\text {......... } \\
\text {-....... } \\
\text {......... }\end{array}$ & $\begin{array}{l}\text { Pkg. } \\
1000 \\
\ldots . . . .\end{array}$ & $\begin{array}{r}.10 \\
.50 \\
. . . . . . .\end{array}$ \\
\hline ona............. & 1 & .50 & .40 & .30 & .25 & .20 & .......... & 10 & .20 \\
\hline
\end{tabular}

\section{RAINBOW COLLECTION}

For beginners and those who do not want to bother with keeping the varieties separate I suggest my Rainbow Collection. This is a collection of 100 large ( 1 inch up, mostly up) bulbs in 30 or more varieties in various shades of red, pink, blue, white, smoke and others. These are all first class varieties and sure to please but are not labelled. I honestly believe this to be the finest collection offered by anyone for the price and better than most collections regardless of price.

100 for $\$ 3.00 ; 50$ for $\$ 1.75$. All prepaid.

If you do not like certain shades I can leave them out and put in more of others.

\section{A Few Testimonials}

"The bulbs I got of you last year gave perfect satisfaction."

"I have never been more pleased with bulbs than I was with those I purchased from you last season and that is saying a great deal for not less than 50 catalogs are sent to me yearly and I have patronized most of them all the way to the west coast."

"The collection I got from you last year was simply superb."

"My bulbs arrived last evening and were in the pink of condition, indeed a very fine lot. I am more than pleased with them and right here please accept my sincere thanks for all the extras you put in on every variety. The more business I do with you the more I want to, not alone because of your generosity but because I believe from past and present experience, in fact I know that you not only do as you agree but much more and if the gladiolus buyers realized your honorable dealings with your customers you could not begin to supply the demand.

The above is from a customer who has sent me several big orders, both last year and this.

"I want to thank you for the beautiful bulbs of Persia you sent me. What do you use for a disinfectant to treat your bulbs"?

If space permitted I could give you many more equally strong testimonials. 